Daimon. Revista Internacional de Filosofía, n ${ }^{\circ} 85$ (2022), pp. 143-159

ISSN: 1130-0507 (papel) y 1989-4651 (electrónico)

http://dx.doi.org/10.6018/daimon.404851

Licencia Creative Commons Reconocimiento-NoComercial-SinObraDerivada 3.0 España (texto legal). Se pueden copiar, usar, difundir, transmitir y exponer públicamente, siempre que: i) se cite la autoría y la fuente original de su publicación (revista, editorial y URL de la obra); ii) no se usen para fines comerciales; iii) se mencione la existencia y especificaciones de esta licencia de uso.

(c) (1) (3)

\title{
Creatividad, paz, educación: algunas relaciones conceptuales*
}

\section{Creativity, peace, education: some conceptual relations}

\author{
ALEJANDRA HERRANZ CASTEJÓN*** \\ JULIO JOSÉ MOYANO FERNÁNDEZ****
}

\begin{abstract}
Resumen: Este trabajo presenta una comparación de la noción de creatividad según Csíkszentmihályi con las concepciones de paz observadas por Galtung, así como de la evolución de estos conceptos. Además, se enfatiza que estas nociones están en concomitancia en tanto en cuanto la creatividad es necesaria como herramienta para la transformación de conflictos de forma pacífica. El nexo que se propone para unir ambos conceptos es la educación, incardinada en un constructo que se denominará triángulo abstractivo.

Palabras clave: Educación, paz, creatividad, triángulo abstractivo.
\end{abstract}

\begin{abstract}
This work presents a comparison of the notion of creativity by Csíkszentmihályi with peace's conceptions observed by Galtung, as well as of the evolution of those issues. Moreover, it is emphasized that both concepts agree in the sense that creativity is necessary as a tool for a peaceful conflict transformation. This agreement occurs in the field of education, and we propose as a framework a theoretical construct called abstractive triangle.
\end{abstract}

Keywords: Education, peace, creativity, abstractive triangle.

Recibido: 28/11/2019. Aceptado: 20/04/2020.

* Este trabajo ha sido parcialmente financiado por el Proyecto de Innovación Educativa núm. 3846, año 2020, dentro de la acción de innovación educativa «Millora de la comprensió lectora lògica-matemàtica» con código 18G002-299, del Grupo de Innovación Educativa IEALYGEO de la Universitat Jaume I de Castelló, al que los autores pertenecen.

** Alejandra Herranz es graduada en Humanidades: Estudios interculturales por la Universitat Jaume I de Castelló; también se encuentra finalizando el Grado en Historia y Patrimonio, así como el Máster en Prevención de Riesgos Laborales en la mencionada universidad. Su trabajo de final de grado en Humanidades se titula Una mirada crítica a la apropiación cultural (2019). Además ha publicado: Herranz, A., MoyanoFernández, J.J. (2018), «Creatividad y crítica en la enseñanza de las matemáticas» en: Arnal Pons, A.M. et al (ed.): Actas del Congreso Virtual: Avances en Tecnologías, Innovación y Desafíos de la Educación SuperiorATIDES 2018, Castelló de la Plana: Publicacions de la Universitat Jaume I, pp. 245-256. Correo electrónico: a1339514@uji.es.

*** Julio José Moyano es Doctor en Matemáticas por la Universidad de Valladolid y Profesor Titular de Universidad en la Universitat Jaume I. Su interés por la reflexión en contextos educativos le ha llevado a publicar: Gregori, P., Martínez, V., Moyano-Fernández, J.J. (2018), «Basic actions to reduce dropout rates in distance learning», Evaluation and program planning 66, pp. 48-52; Herranz, A., Moyano-Fernández, J.J. (2018), «Creatividad y crítica en la enseñanza de las matemáticas» en: Arnal Pons, A.M. et al (ed.): Actas del Congreso Virtual: Avances en Tecnologías, Innovación y Desafíos de la Educación Superior-ATIDES 2018, Castelló de la Plana: Publicacions de la Universitat Jaume I, pp. 245-256. Correo electrónico: moyano@uji.es. 


\section{Introducción}

«Creatividad» es una palabra muy presente en la sociedad actual; es ciertamente una necesidad fundamental del ser humano, cuya satisfacción permite alcanzar una mayor calidad de vida. Ser creativo ha entrado incluso a formar parte de las agendas políticas de los países desarrollados, llegando a considerarse su implementación en I+D+i. Dado que los gobiernos dedican muchos recursos a la educación, parece natural preguntarse: ¿qué presencia tiene la creatividad en el sistema educativo vigente? Un contexto educativo específico en el que juega un papel importante es la perspectiva adoptada en los llamados Estudios de la Paz, en los que se necesitan planteamientos creativos (Benito, García, 2001, p.65).

En este trabajo se pretende elaborar un marco de referencia centrado en la relación de la creatividad con los Estudios de la Paz que oriente a enfocar la Educación para la Paz o educación para la «cultura de la paz» a un nivel definitorio, esto es, estableciendo ciertas relaciones a partir de su significado y su evolución histórica.

La Educación para la Paz se puede situar dentro de un contexto de Filosofía para la Paz que, aunque hunde sus raíces de manera genérica en la época de la Ilustración-de forma clara en Kant, que expone la realización de la idea de un orden cosmopolita basado en la federación de los pueblos en su obra «La paz perpetua»-, recibe un primer impulso tras el desastre de las dos guerras mundiales, y un segundo impulso tras el fin de la Guerra Fría y la aparición de nuevas formas globalizadas de violencia, como profundizaremos después.

El objetivo de este estudio es tratar de construir un esquema conceptual en el que se puedan describir propuestas efectivas a la hora de compatibilizar la teoría de la creatividad con la Educación para la Paz, en el que se reconozcan y encajen algunas habilidades esenciales para la inserción del concepto de paz en el aula. Este esquema lo llamaremos triángulo abstractivo y conjuga la clasificación de los tipos de violencia (directa, estructural, cultural) y sus correspondientes tipos de paz (paz negativa, paz positiva, cultura de paz) según el sociólogo y matemático Johan Galtung $(1969,1990)$ con la definición de creatividad propuesta por el psicólogo Mihály Csíkszentmihályi (1996).

El trabajo se estructura de la manera siguiente: En la Sección 2 se presentan los conceptos de paz y Educación para la Paz. La Sección 3 discute diferentes visiones de la noción de creatividad, justificando la elección de aquella que se toma como central para este trabajo. La Sección 4 sintetiza los conceptos anteriores en el constructo que se denomina triángulo abstractivo. Se cierra el presente análisis con un apartado de conclusiones.

\section{Educación para la Paz}

Esta sección trata de esclarecer uno de los conceptos clave de este estudio. Se concibe la Educación para la Paz como una educación que contemple herramientas para la promoción de una cultura de paz.

2.1. Paz: consideraciones históricas. Los Estudios sobre la Paz y la Educación para la Paz no son lo mismo: los primeros conforman una disciplina académica surgida tras la Segunda Guerra Mundial, mientras que la segunda ha sido contemplada por generaciones desde siempre, pues las sociedades han deseado vivir pacíficamente (Harris, 2002, pp.17-18). 
Históricamente se puede pensar en una primera etapa en la que los conceptos de guerra y paz no se asumen, sino que se aceptan como parte integrante del día a día, en lo que podríamos llamar un concepto de paz vacía: guerra y paz no se diferencian de otros quehaceres cotidianos, no se cuestionan. Sin embargo, a medida que el ser humano se va civilizando, va tomando conciencia de sus posibilidades como ser creativo, y busca vivir en paz para desarrollarse. Se sigue viendo la guerra como necesaria, pero se valora la paz (en lo que se podría denominar paz negativa siguiendo a Galtung, como luego veremos).

Así, va naciendo una conciencia de paz en la historia del pensamiento; los educadores y filósofos se empiezan a preocupar por la paz: ya se ha citado a Kant, pero un siglo antes, Comenio pensaba que «debe desterrarse de la educación toda violencia, por contraria a la naturaleza. Omnia sponte fluant, absit violentia rebus; tal es la divisa disciplinaria de Comenio» que se entiende como «método vivo capaz de actuar verdaderamente sobre el alumno» (Piobetta, 2017, p.138). Comenio tiene presente la Guerra de los Treinta Años; son justo las experiencias bélicas o los aires revolucionarios que se irán sucediendo en Occidente lo que mueva a muchos pensadores a posicionarse: desde los contractualistas, como Locke (para quien el ser humano es libre, la experiencia es el punto de partida y el alumno es una tabula rasa al comenzar) o Rousseau (que considera al hombre bueno por naturaleza y a la educación como su apoyo para convivir en un mundo corrupto), hasta el pragmatista John Dewey, la celebérrima Maria Montessori o la pedagogía crítica de Paulo Freire, por ejemplo. De hecho, el siglo XX nos presenta tres tradiciones que terminan confluyendo en la Educación para Paz: la tradición de la educación libertaria iniciada por Charles Fourier (1772-1837) y Pierre-Joseph Proudhon (1809-1865), la del desarrollo personal y la de la educación para la comprensión internacional (Hicks, 1993, pp.23-24).

Por otro lado, la pretensión de encontrar las formas más pacíficas posibles que hagan más justas las relaciones injustas anida en el núcleo de los llamados Estudios en Investigación sobre la Paz (Brunk, 2000, p.25). El desarrollo de estos estudios a nivel institucional se produce después de la Segunda Guerra Mundial, con dos focos: Michigan, con Kenneth Boulding y Anatol Rapoport a la cabeza, y Escandinavia, impulsado por Johan Galtung. Aquí destacaremos la teoría de Galtung, en la que distingue tres tipos de violencia a los que contrapone tres nociones de paz:

o Una violencia directa o individual, que es física, visible, patente en forma de conductas, de forma que es el individuo quien puede asumir, o asume, la violencia directa en su comportamiento, pero está, al menos teóricamente, en su mano rechazarla (noción de paz negativa).

o Una violencia indirecta o estructural, invisible; la negación de las necesidades del individuo, que no son satisfechas en el marco en que este actúa, deviene en un tipo de violencia estructural, que se puede contrarrestar con la construcción de puentes hacia un concepto de paz positiva.

o Una violencia cultural, igualmente sutil. La cultura en que está envuelto el individuo crea un marco que viene a legitimar la violencia y posibilita desarrollos actitudinales en la generación de esta; es el desarrollo de una cultura de paz lo que se puede oponer a esta forma de violencia. 
Estos conceptos de violencia y paz se representan en sendos triángulos, que llamaremos triángulos de Galtung (Galtung, 1998, p.15):
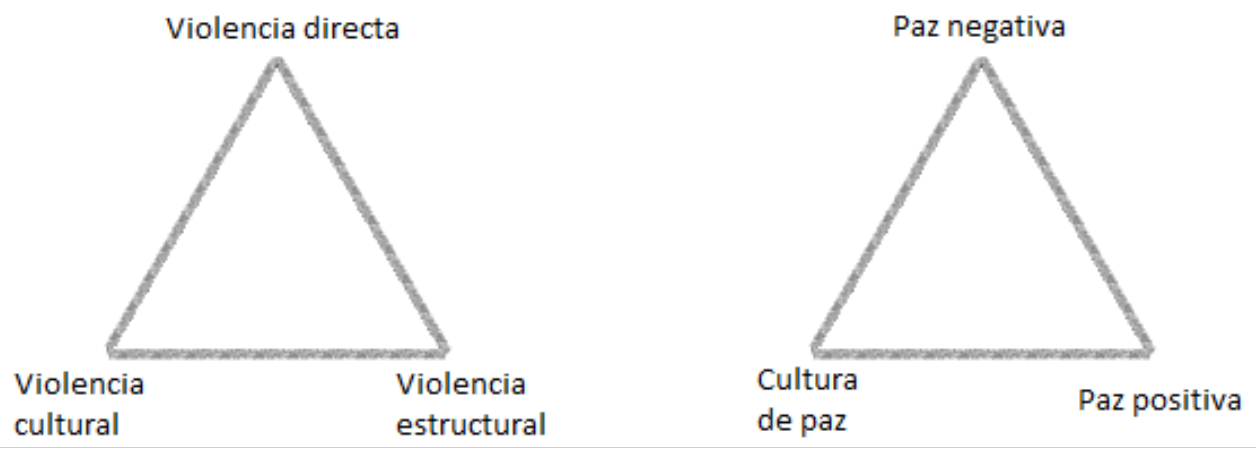

Ilustración 1. Triángulos de Galtung

A la luz de la teoría galtungeana y la exposición previa, se puede concluir que la humanidad distingue cuatro etapas en el desarrollo del concepto de paz, resumidas en la Tabla 1.

\begin{tabular}{|c|c|c|}
\hline Etapa 1 & Paz vacía & No consideración del concepto \\
\hline Etapa 2 & Paz negativa & Ausencia de guerra \\
\hline Etapa 3 & Paz positiva & Ser humano como agente \\
\hline Etapa 4 & Cultura de paz & Influencia del entorno y estructuras \\
\hline
\end{tabular}

Tabla 1. Etapas del concepto de paz

2.2. Filosofía de la Paz y educación. En la Sección 2.1 se ha visto cómo algunos filósofos parten de presupuestos en términos de paz (o libertad, o bondad, o igualdad). El desarrollo de los Estudios de la Paz impulsa a su vez una Educación para la Paz, desde una perspectiva multidisciplinar, con aportaciones sociológicas, antropológicas o psicológicas, y la filosofía como telón de fondo. Sin embargo, ha de abogarse por un verdadero diálogo que conecte tales aportaciones, esto es, interdisciplinariedad. Tal diálogo ha de trascender las humanidades y consolidarse junto a las artes y las ciencias por medio de la creatividad como nexo herramental al servicio de la Educación para la Paz, pues la creatividad es un proceso en que ciencias y humanidades tienen que ir de la mano, como apunta E. O. Wilson (2018, p.81): «Science and humanities share the same origin and brain processes of creativity». Este punto de encuentro entre ciencias y humanidades que es la creatividad, es señalado por Galtung como parte del camino hacia la paz, donde entiende paz como «capacidad de manejar los conflictos con empatía, no violencia y creatividad» (Galtung, 1998, p.18). Pero ¿cómo entender la creatividad?

Se comprende, por todo lo expuesto, que tal creatividad haya de partir de la meditación de postulados filosóficos, de una reconsideración de los puntos de partida y de un análisis 
de la situación actual de los conceptos y tendencias, con visión histórica; en tal sentido se podría hablar de una creatividad de índole filosófica.

\section{Creatividad}

Tras una clarificación de los conceptos de paz y de Educación para la Paz, como un intento de refuerzo de esta última se ha propuesto un diálogo interdisciplinar que cuentecomo elemento cohesionador-con la idea de creatividad apelada desde la reflexión filosófica. En la presente sección se concreta este concepto. En efecto, la creatividad no se puede reducir a un mero proceso constructor, pues se comprende como un rasgo distintivo, definitorio, de la especie humana: «La mayor parte de la investigación sobre la creatividad se ha centrado o bien en sus mecanismos cerebrales, o bien en los factores sociales y culturales que la afectan» (Goldberg, 2019, p.10).

Ahora bien, ¿qué se entiende por creatividad? ¿Qué nociones de creatividad se contemplan? Es imprescindible detenerse en el significado de esta noción, pues se constatan algunos equívocos cuando se habla de creatividad: ni es sinónimo de innovación, aunque la pueda implicar, ni es equivalente a la adaptación a los nuevos tiempos, esto es, modernización, aunque pueda ser un motor de ésta. Además, escudriñando la evolución histórica de su significado, se dará cuenta de un curioso paralelismo con la visión de la noción de paz que se ha venido considerando a lo largo de la historia.

3.1. Evolución histórica. Una primera observación, crucial, es que este término es relativamente nuevo en las lenguas europeas. La palabra «crear» estaba reservada a $\operatorname{Dios}^{1}$ : el ser humano no creaba. Aunque no son solamente reminiscencias de la tradición cristiana, pues por ejemplo en la Antigüedad no hay reflejo de tal uso; más aún, Platón no sentía ninguna simpatía por sus coetáneos creadores: se refiere en la República (607b) a «una antigua desavenencia entre la filosofía y la poesía» y quería desterrar a los poetas, esto es, los artistas. Platón consideraba la poesía como «una actividad creadora en general», entroncando con el sentido primigenio de poíesis, que se deslizó desde el «hacer, fabricar, producir, edificar» en general hasta el «crear», «representar algo o representar a alguien (artísticamente)» y «crear algo con la palabra». La poesía sería entonces el proceso de tal creación (Lledó, 2010, pp.19-21, p.43). Aunque precursor, este no es el sentido de la creatividad tal y como se entiende hoy en día, ya que en aquel tiempo el creador, el artista, el poeta, seguía siendo tutelado por los dioses, por el dictado de las musas.

Si bien en los Diálogos de Nicolás de Cusa ya se atisba una ruptura entre la concepción platónica de idea y la noción humanística de invención ${ }^{2}$, podemos decir que la creatividad en su sentido actual nace con la Ilustración. Hablar de creatividad exige un presupuesto filosófico de novedad, un ex-nihilo, que en cierta medida se opone a la noción de descubrimiento. Desde este punto de vista, la creatividad no tiene cabida en ninguna teoría platónica. El primer con-

1 Como lo atestigua el DRAE en su XXIII edición - o así se puede interpretar-cuando, como primera acepción de crear, nos dice que es producir algo de la nada.

2 Nicolás de Cusa ofrece un interesante diálogo de un escolástico frente a un artesano, en el que este le arguye a aquel que la forma de las cucharas que produce son obra suya y de ningún modo imitación de la naturaleza. 
cepto que se tiene en cuenta es el de imaginación ${ }^{3}$, como hacen, por ejemplo, Kant o Hobbes, o el de la fantasía, como los idealistas alemanes, quienes veían en la originalidad y en un ilimitado poder creativo la esencia de la subjetividad libre (Taylor, 2010, p.40). Hegel menciona en El primer programa de sistema del idealismo alemán: «La primera idea es naturalmente la representación de mí mismo como un ser absolutamente libre. Con el ser libre, consciente de sí mismo, brota a la vez de la nada un mundo entero, la única creación verdadera y pensable desde la nada», citado en (Safranski, 2018, p.76). La fantasía posibilitaba así

la unidad entre subjetividad y naturaleza. [...] La razón era vista como facultad divisiva, diseccionante, analítica, una que sólo podía alejarnos de la unión con la naturaleza. [...] la unidad del consciente y el inconsciente permanecería como algo no completamente comprendido. [...] El asunto sobre la creación artística, como lo estableció Kant, es que no podemos dar su fórmula. (Taylor, 2010, p.41)

Y sin embargo no se ha cejado en el empeño de encontrar tal fórmula. La introspección se localizó pronto como fuente de conocimiento para descubrir los entresijos de la creatividad y fue practicada por muchos científicos que intentaron explicar la consecución de la idea feliz. El objetivo perseguido, ayer como hoy, es intentar deducir una ley de validez general que nos proporcione ideas felices con las que alimentar la ciencia y el progreso; sin duda una ocurrencia solamente posible en el seno del concepto moderno de ciencia. Este disfrute creador antropocéntrico, con el que el ser humano se dota de la libertad de creación, hace emerger la idea del genio, que impregnará la época del romanticismo alemán, con todas sus consecuencias: «la filosofía de la vida de Herder estimuló el culto al genio en el movimiento Sturm und Drang y más tarde en el Romanticismo. En ellos se considera genio a aquel en quien la vida brota con libertad y se desarrolla con fuerza creadora» (Safranski, 2018, pp.22-23).

Ha habido intentos de una detallada descripción del proceso creativo. El matemático William Hamilton legó sus experiencias, pero de una forma tan particularizada que no tuvieron demasiada acogida. Diferente fue el célebre «La invención matemática» de Jules Henri Poincaré, quien logra una descripción estructurada del proceso de invención, aunque aún en forma primitiva (Poincaré, 2018). Se vislumbran así cuatro etapas del proceso creador que, como señaló Jacques Hadamard (1954), podrían denominarse trabajo preliminar, incubación, iluminación y verificación. Grahan Wallas, cofundador de la London School of Economics, añade a estas una quinta etapa: entre incubación e iluminación sitúa la intimación o ese sentimiento íntimo que hace sentir al creador que está en la buena dirección y le anima en el empeño, (Wallas, 1926). En este estado de cosas, Alfred N. Whitehead, en una serie de conferencias, acuña el término «creatividad» en el sentido que nos ocupa, como señala Steven Meyer:

Whitehead actually coined the term-our term, still the preferred currency of exchange among literature, science, and the arts... a term that quickly became so popular, so omnipresent, that its invention within living memory, and by Alfred North Whitehead of all people, quickly became occluded. (2005, pp.2-3)

3 Un libro referente sobre creatividad debido a Osborn y publicado en 1953 tiene todavía por título Applied Imagination. 
Nótese que estos autores estaban fuertemente influidos por la filosofía vitalista de Bergson, quien proclamaba que la vida «is invention, is unceasing creation» (2005, p.27), arrastrada por el célebre impulso vital. Aunque puede que la influencia vitalista tenga poco que ver. Tomando en consideración a Wittgenstein, el filósofo de la destrucción si nos atenemos a sus palabras «It came into my head today as I was thinking about my philosophical work and saying to myself: „I destroy, I destroy, I destroy - “» (1980, p.21), también su círculo cercano analizaba sus fases de creación. Como citan Janik y Toulmin, Rudolf Carnap lo describía así:

La postura y actitud que adoptaba ante la gente y los problemas, aun los teóricos, se parecían mucho más a las de un artista creativo que a las de un científico; casi se podría decir que eran similares a las de un profeta o un vidente. [...] No es que expusiera sus opiniones de manera dogmática... pero nos causaba la impresión de que llegaba a un entendimiento súbito como producto de una inspiración divina, de modo que no podíamos evitar sentir que cualquier comentario o análisis sensato y racional que hiciéramos al respecto constituiría una profanación. (2017, p.16)

Carnap confiere un halo verdaderamente místico a Wittgenstein en su proceso creativo. Sin embargo, parece eludir un sentido de profanación que, como advierte el jurista romano Gayo Trebacio Testa, «es aquello que, habiendo sido sagrado o religioso, es restituido al uso y a la propiedad de los hombres» (Fernández Mallo, 2018, p.335), para conferir un nuevo sentido a lo profanado. Esta visión, empero, entra en una dimensión más bien psicológica de la creatividad que, bajo la influencia de la obra de Freud, acaparó una buena parte de la discusión en los años siguientes; precisamente fue G. P. Guilford, presidente de la American Psychological Association en 1950, quien contribuyó a la generalización del término «creatividad» (Barrena, 2007, p.19). Tras este período, los trabajos se centraron en la comprensión de los mecanismos a nivel biológico: más que describir la creatividad, se intenta entenderla. La idea romántica, como se ha visto, de creatividad ligada a la de genialidad sigue presente a mediados de los años 50, también en los ámbitos pedagógicos, cuando todavía se suponía que solamente el alumnado bien dotado era susceptible de ser creativo.

Sin entrar a analizar las causas ${ }^{4}$, puede decirse que esta situación ha cambiado por completo. Actualmente se aboga por una comprensión de la interacción de esta dualidad biología-sociedad, como se trata en Goldberg (2019), que además intenta interconectar diversos aspectos extrínsecos al individuo. Por su vigencia, se podrían citar aquí los trabajos de Mihály Csíkszentmihályi, de algún modo interpretados por Angélica Sátiro (2002), de Teresa Amabile (2000) y de Ken Robinson (2009). Los dos primeros autores se centran en el llamado Modelo de Sistemas, que enfocan su atención no solamente en la persona, sino también en el campo (aspectos culturales) y el ámbito (aspectos de organización social del campo). Amabile (2000, p.8) formula el principio de la creatividad por motivación intrínseca, que afirma que «las personas alcanzarán su máximo grado de creatividad cuando se

4 Si suponemos que esta idea es heredada de la concepción romántica basada en el idealismo alemán, bien pudiera relacionarse la desaparición de esta concepción con el declive de Alemania como potencia intelectual tras el nazismo. 
sientan motivadas fundamentalmente por el interés, satisfacción y reto del trabajo en sí y no por presiones externas». En cuanto a Robinson, propone la existencia de un elemento, es decir, aquella actividad por la que el ser humano se abstraería de entusiasmo, de pasión, hasta llegar incluso a olvidar el sentido del tiempo.

Sintetizando, en la exposición histórica anterior se pueden observar cuatro etapas en la consideración social de la creatividad, al menos en Occidente:

\begin{tabular}{|l|l|l|}
\hline Etapa 1 & Creatividad vacía & No se contempla el acto de creación en sí \\
\hline Etapa 2 & Creatividad dada & Dios crea; y crea de la nada \\
\hline Etapa 3 & Creatividad genial & El poder de la fantasía creadora está dentro del ser humano \\
\hline Etapa 4 & Creatividad estructural & Es el resultado de múltiples factores internos y externos al \\
ser humano
\end{tabular}

Tabla 2. Etapas del concepto de creatividad

Las etapas de la Tabla 2 se pueden situar cronológicamente más o menos así: la Etapa 1 duraría hasta la inclusión en el lenguaje de palabras específicas para el acto de creación, la Etapa 2 hasta la época ilustrada, la Etapa 3 hasta las investigaciones psico-sociológicas del siglo XX y la Etapa 4 desde ese momento hasta la actualidad. Estas etapas pueden, además, relacionarse con las cuatro etapas de la Tabla 1: en ambas tablas existe una primera etapa en la que los conceptos en cuestión no se piensan como tales, sino que se asumen como dados o como parte del mundo; una segunda etapa en la que el concepto ya se ha asumido como tal, pero sobre el que no parece ser posible ejercer ningún control: en el caso de la paz, es la ausencia de la guerra, que a su vez se ve como recurso necesario, y en el caso de la creatividad, viene de Dios; se sucede una tercera etapa en la que es el ser humano quien se empieza a reconocer como actor y regulador de los procesos, y una cuarta etapa en la que hay un reconocimiento de la complejidad de los procesos, en la que se examina una influencia de agentes externos junto a la consideración de estructuras asociadas. A la vista de este paralelismo, no puede extrañar que se trate de profundizar en la relación entre los conceptos de paz y creatividad.

3.2. La creatividad como tríada. De los conceptos de creatividad de Csíkszentmihályi, Sátiro, Amabile y Robinson referidos en el apartado anterior, estimamos pertinente tomar el sentido dado por Csíkszentmihályi a la creatividad. Descartamos las otras tres en nuestra aproximación por diversas razones: la interpretación de Sátiro es, en su esencia, demasiado cercana a la de Csíkszentmihályi, por lo que nos quedamos con la original. Amabile basa su punto de vista en otro de dificultad equivalente, el de motivación, por lo que, a nuestro juicio, no hace sino trasladar la problemática de la creatividad a la de la motivación. Robinson, por otra parte, nos parece demasiado cercano a esa ideología del «pensamiento positivo» que pretende instalar al mundo en un buenismo histriónico, sumergir a la sociedad en la creencia de que los pensamientos cambian necesariamente 
la realidad: una evidencia se puede leer ya en el subtítulo de su libro El Elemento (2009, portada): «Descubrir tu pasión lo cambia todo».

Justificados los motivos de descarte de otras propuestas coetáneas, se toma la de Csíkszentmihályi (1996, p.6):

Creativity results from the interaction of a system composed of three elements: a culture that contains symbolic rules, a person who brings novelty into the symbolic domain, and a field of experts who recognize and validate the innovation. All three are necessary for a creative idea, product, or discovery to take place ${ }^{5}$.

Esta definición de creatividad no solamente asume la habilidad (de indagación y problematización) del individuo, sino también una dependencia tanto de la cultura donde se desenvuelve como del reconocimiento de la comunidad. En definitiva, puede ser entendida como un valor cultural que permite generar soluciones eficaces para las problemáticas contemporáneas, basado en un pensamiento reflexivo, flexible y divergente; es una solución independiente y autónoma de los problemas, aunque aprobada por la comunidad. Se puede describir por medio de un triángulo, que llamaremos triángulo de la creatividad:

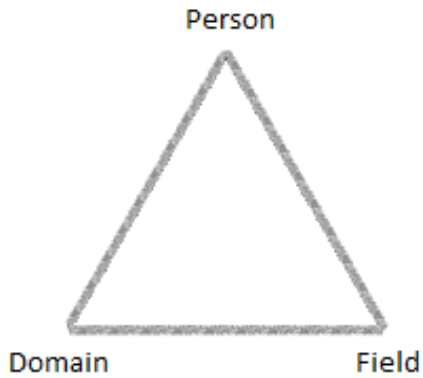

Ilustración 2. Triángulo de la creatividad

En él, los vértices corresponden a las tres vertientes expresadas en la definición de Csíkszentmihályi, es decir:

- la persona (person) como motor de la creación, quien toma la iniciativa en el proceso creador: «To be creative, a person has to internalize the entire system that makes creativity possible» (Csíkszentmihályi, 1996, p.51);

- el ámbito o dominio (domain), es decir, la parcela de conocimiento en la que se ejerce la innovación. Si se desea, se puede entender como el conjunto de reglas y procedimientos; por ejemplo, la física, o, si se quiere hilar más fino, la termodinámica o la física cuántica son ejemplos de ámbitos;

5 «La creatividad resulta de la interacción de un sistema compuesto por tres elementos: una cultura que contiene reglas simbólicas, una persona que aporta novedad en el dominio simbólico, y un campo de expertos que reconozcan y validen la innovación. Todos ellos resultan necesarios para que se produzca una idea, producto o descubrimiento creativo». (Traducción de los autores). 
- el campo (field), necesario para determinar «whether the innovation is worth making a fuss about» (Csíkszentmihályi, 1996, p.41). Incluye a todos los individuos cuya misión es decidir si una nueva idea o producto debe ser incluido en el ámbito y, con ello, entrar a formar parte de la cultura. Son ejemplos de campos las agencias gubernamentales que tratan de la cultura, directores de museos, referees de revistas de investigación, críticos de arte, etc.

Persona, ámbito y campo son tres aspectos relacionados: «the trait of personal creativity may help generate the novelty that will change a domain, but it is neither a sufficient nor a necessary condition for it» (Csíkszentmihályi, 1996, p.29). De hecho, la creatividad en el aquí y el ahora no depende solamente de lo creativo, en términos cuantitativos, que sea un individuo, sino que «it depends just as much on how well suited the respective domains and fields are to the recognition and diffusion of novel ideas» (Csíkszentmihályi, 1996, p.31).

Esta definición no está exenta de crítica. Por ejemplo, Barrena afirma: «Se plantea por supuesto la cuestión de quiénes son los expertos y si puede haber expertos para algo que precisamente se caracteriza por la novedad» (2007, p.21). La segunda cuestión se puede resolver dentro de la propia teoría, pues no se quiere decir, a nuestro modo de ver, tanto que se trate de expertos en el tema concreto que se propone como de árbitros con experiencia dentro del campo de conocimiento en el que se desarrolla la propuesta candidata a ser creativa. Sí se puede encontrar un escollo en la primera cuestión mencionada, sobre todo si no se hace una hipótesis de buena voluntad sobre el comité de expertos. Desde otra perspectiva, «desde el momento en que el autor propone la intervención del subsistema cultura se enfrenta a una situación de no fácil resolución» (Pascale, 2005, p.82), por cuanto que el concepto de cultura entraña múltiples dificultades conceptuales; claro que esta idea no es novedosa: se trata de la celebérrima observación wittgensteiniana de la imposibilidad de definir ciertos conceptos solamente con condiciones necesarias y suficientes, por lo que se precisa recurrir a la noción de «Familienähnlichkeiten» (Wittgenstein, 1988, p.87).

Sea como fuere, la definición de creatividad propuesta por Csíkszentmihályi sintetiza ciertas nociones asociadas al proceso de invención que emana del método científico. Por ejemplo, lo que Jorge Wagensberg (2019, pp.215-218) reconoce como los tres conceptos fundamentales del método científico, a saber, realidad, observación y comprensión, encuentran sustento teórico en el esquema de Csíkszentmihályi: efectivamente, la observación o percepción de la realidad para su comprensión, es decir, para la comprensión de la observación, si no de la realidad misma, tiene una lectura en clave triádica. Es el sujeto quien percibe por diferenciación de aspectos observados en un mismo entorno, un campo, que es la realidad, con el propósito de comprenderla, buscando similitudes en las diferentes observaciones de la realidad, y por tanto, juzgando y analizando, esto es, erigiéndose en su propio árbitro del conocimiento. En este sentido, se podría decir que la visión de Csíkszentmihályi es una extensión del método científico propuesto por Wagensberg, o que este es una restricción al sujeto de aquel. Tampoco es una observación del todo sorprendente, teniendo en cuenta que Csíkszentmihályi está influido por la propuesta epistemológica llamada Teoría General de Sistemas debida a Von Bertalanffy (Pascale, 2005, p.79).

Esta estructura triádica, unida a la tríada persona-ámbito-campo de Csíkszentmihályi, es una reinterpretación de la tipología que ofrecen los triángulos de Galtung. En la sección 
siguiente se explicarán las conexiones entre creatividad, paz y la mencionada tríada, sintetizada en la construcción del triángulo abstractivo.

\section{Creatividad y paz. El triángulo abstractivo}

Esta sección aborda el vínculo entre creatividad y paz a través de la educación. Las consideraciones históricas sobre la creatividad expuestas en el apartado anterior permiten deducir algunas observaciones:

- La descripción del proceso creativo se suele asociar con experiencias agitadas, destructivas, más que con pacíficas meditaciones; parece darse a entender un predominio de lo dionisíaco. Hay un elemento violento en el proceso creativo, que algunos sienten como si fuera un rayo, otros como una agitación extenuante, etc.

- Se tiende a establecer una relación entre la creación humana como reflejo de la divina, bien patente por ejemplo en la descripción de Carnap sobre Wittgenstein en la Sección 3.1. Ello podría querer decir que los patrones de referencia aún toman la creación divina como paradigmática, es decir: existe un elemento extrínseco en el proceso creador (cosa que se reconoce en algunas definiciones de creatividad) que trasciende el propio proceso (he aquí el hecho diferenciador).

- Se compara el creador con el artista, como si fuera este el paradigma del creador. Es decir, la obra de arte es la creación por excelencia en el imaginario colectivo, lo que podría verse como un reduccionismo en cuanto a la vinculación de la creatividad con el mundo: el ojo con el que un artista ve el mundo y lo interpreta no ha de ser necesariamente parecido al ojo con el que lo hace un físico, póngase por caso.

Estas observaciones hacen, en principio, lejana una comparación entre el proceso creativo y la paz como creación humana, ya que:

- La creatividad se relaciona con lo dionisíaco, en tanto que la paz estaría en consonancia con lo apolíneo.

- Tanto la cultura de paz como la paz positiva se entienden solamente situando al ser humano como agente constructor de los procesos.

- La paz no se puede restringir a un ámbito, con sus condicionantes característicos, si hablamos de paz positiva.

Sin embargo, los puntos de encuentro entre creatividad y paz expuestos en apartados anteriores, englobados en la creatividad de índole filosófica, se juzgan motivados por el devenir histórico de la humanidad: Tras dos guerras mundiales, y ante la Guerra Fría-la división del mundo en dos bloques antagónicos, enemigos, y potencialmente aniquiladores- ¿qué esperanza le quedaba a la humanidad? Muchos vieron la respuesta en la educación. Pero ¿de qué modo enfocar la educación después de Auschwitz? Se mencionan dos autores que ofrecen respuestas interpretables como síntesis de la relación entre paz y creatividad en la educación que se propugna en este estudio. El primero, Theodor W. Adorno, contestaba así:

Cuando hablo de la educación después de Auschwitz hablo de dos ámbitos: en primer lugar, educación en la infancia [...]; seguidamente, ilustración general llamada a crear un clima espiritual, cultural y social que no permita una repetición. (1998, p.81) 
La Ilustración es entendida en general por la Escuela de Fráncfort como progreso, presidido por el afán de la persona de madurar como ama de la naturaleza externa e interna ${ }^{6}$, lo cual implica una postura generalista, del ser humano como en permanente necesidad de educación, sumido en esa ambigüedad que encierra para Adorno y Horkheimer la Ilustración: «quiere liberar a los hombres del miedo y constituirlos en señores» $(2009$, p.59) pero dominando la naturaleza. Esta liberación por dominio es el punto crítico, pues «la naturaleza [se disuelve] en mera objetividad» $(2009$, p.64). Por ello, la ilustración general que desea Adorno apela a la creatividad para instaurar un nuevo orden espiritual y sociocultural que se defienda de la propia Ilustración.

Adorno observó ciertamente que el mal alcanzaba una nueva dimensión, como también supo reconocer Hannah Arendt:

The most conspicuous and most dangerous fallacy in the proposition, as old as Plato, «Nobody does evil voluntarily», is the implied conclusion, «Everybody wants to do good». The sad truth of the matter is that most evil is done by people who never made up their mind to be either bad or good. (1971, p.438)

Arendt es, posiblemente, una de las pensadoras más influyentes en cierta reconfiguración de la idea de la presencia del mal en el mundo. En su opúsculo Sobre la violencia reflexiona sobre el carácter instrumental de la violencia y su relación con el poder y la autoridad, aunque desde el punto de vista de la teoría política. El poder, según Arendt, «corresponde a la capacidad humana no simplemente para actuar, sino para actuar concertadamente. El poder pertenece a un grupo y desaparece con él» (2015,p.108). Poder y violencia no son lo mismo, pero existe una tradición de pensamiento que tiende a asimilarlos. De esta interpretación «tradicional» de poder destaca Arendt, citando al politólogo y economista francés Bertrand de Jouvenel (1903-1987), que es creativo porque «le es propio el instinto de crecer». En él observa un «impulso interno de crecimiento» (2015, p.129). Por ello, las revoluciones estaban dirigidas contra los poderes establecidos «solamente desde un punto de vista exterior», y tenían la capacidad de imprimir en el poder «un nuevo vigor y un nuevo equilibrio, y derribar los obstáculos que habrían obstruido durante largo tiempo su desarrollo» (2015, pp.129-130). Continúa Arendt: «Cuando Fanon ${ }^{7}$ habla de la 'locura creativa' presente en la acción violenta, sigue pensando en esta tradición». Efectivamente, en el arte de la guerra la inventiva ha sido esencial, ayudada por los avances tecnológicos en tiempos más recientes: en táctica, en armamento, en logística, incluso en relaciones internacionales. Por no mencionar también la idea de que la violencia ha sido tomada, en sí misma, como creatividad, en una línea que propugna la necesidad de destruir para construir un mundo o una sociedad mejores. Si se ha aplicado la creatividad en la acción violenta, y si ahora la filosofía ha vuelto los ojos al concepto de paz como «paz positiva», entonces llega el momento de aplicar la creatividad en la construcción de la paz. La pregunta es cómo.

Una respuesta se puede encontrar en el Modelo de Sistemas, al ser «capaz de captar las dimensiones extra personales de la creatividad y armonizarlas en una interacción que expone

6 Cortina (2008: 209).

7 Frantz Fanon (1925-1961) fue un psiquiatra y pensador caribeño, cuyas ideas sobre la descolonización y la psicopatología de la colonización influyeron en el panorama revolucionario de los años 60 y 70 del siglo XX. 
el proceso creativo como una producción atravesada por elementos históricos, culturales, institucionales, sociales, económicos y psicológicos» (Pascale, 2005, p.84); esta perspectiva de la creatividad sugiere un paralelismo con la noción de paz vista como sistema, y no solamente como proceso. La consideración de la paz como sistema está en cierta medida implícita en los triángulos de Galtung: la paz es el resultado de la interacción de unas reglas simbólicas que se condensan en el concepto de cultura, una persona que actúa y un ámbito en el que la cultura en la que está inmersa esa persona se ha dotado de unas normas de moderación o arbitraje.

Por otro lado, es posible dar una respuesta más pragmática a la cuestión de la aplicación de la creatividad a la construcción de la paz, a partir de las definiciones de paz y de creatividad ya expuestas, de la mano de la Educación para la Paz, puesto que:

Se ha conocido así en los últimos años un interés renovado por la enseñanza en el aula de materias controvertidas [...] Es importante desafiar abiertamente algunos de los valores negativos subyacentes a nuestra sociedad, como la aceptación tácita de la violencia masculina, el racismo institucionalizado o la insistencia en un perpetuo consumismo. [...] Para diferentes ocasiones resultarán adecuados distintos enfoques. [...] La educación para la paz constituye un campo en el que se trata claramente de captar esta problemática de un modo creativo e imaginativo. (Hicks, 1993, pp.28-29)

Este fragmento propone la Educación para la Paz como un lugar donde diferentes visiones se aúnan para enfrentarse con los desafíos contemporáneos: tratamiento de las fake news en la era de la posverdad, el auge de los populismos, la revitalización de la xenofobia, etc. Es en esta búsqueda de un tratamiento común para todos ellos donde encuentra la creatividad su razón de ser.

Creatividad y educación son, pues, dos conceptos relacionables; aunque se tiende a pensar en la educación como un mero sistema de aprendizaje, basado en los conocimientos preexistentes, es más que eso: es un sistema que alimenta las dos primeras fases del proceso creativo. Recíprocamente, la creatividad puede ser un motor del sistema educativo (debe serlo en un sistema educativo que se precie de querer innovar). Por otro lado, también tiene sentido pensar en una relación entre educación y paz. Existe, como se insinuaba en la introducción, una corriente de investigación que basa la respuesta en la Filosofía para hacer las paces, Martínez Guzmán (2001), y empieza a buscar referencias en la pedagogía liberadora de Freire (1970), por ejemplo, analizando sus analogías con la llamada comunidad de indagación filosófica propuesta por Matthew Lipman (1988).

Nuestra respuesta a la pregunta formulada sobre cómo aplicar la creatividad en el ámbito de la Educación para la Paz no incide en métodos específicos, sino que consiste en un constructo teórico, en el que se propone un esquema globalizador (que denominamos triángulo abstractivo) para articular al mismo tiempo las visiones aportadas por los triángulos de la creatividad y de Galtung.

El triángulo abstractivo. La definición del triángulo parte de la hipótesis siguiente: Que la creatividad juega un papel importante (en mayor o menor medida) en la paz y más específicamente en la Educación para la Paz, es posible basarlo en la existencia de un paralelismo entre el 
triángulo de la creatividad y los triángulos de Galtung. De hecho, ambos se pueden condensar (abstraer) y considerar un triángulo abstractivo ${ }^{8}$ con, digamos, vértices A, B y C, donde

- al vértice A se le asignan propiedades del individuo, atribuibles a él o acciones de las que él pueda ser sujeto agente o paciente, esto es, propiedades individuales o de la subjetividad;

- el vértice B señala etiquetas de la complejidad y variedad del entorno cultural en el que el individuo desarrolla su acción; en otras palabras, cualidades relativas a la multisubjetividad;

- al vértice $\mathrm{C}$ se le asignan características que relacionan al sujeto con su entorno social, al nivel que se esté tratando, o que tienen que ver con ellas; es decir, propiedades de la intersubjetividad.

Este triángulo permite describir claramente la vinculación entre los triángulos de Galtung y de la creatividad:

- El vértice A relaciona la violencia individual, la paz negativa, con la persona agente de la creatividad. Es el individuo quien ha de tener la voluntad de crear un clima de «no guerra», de cese de lucha, e incluso de querer ser creativo para lograrlo. Una condición necesaria para la paz negativa es, sin duda, la motivación individual para alcanzarla: es la «persona» quien debe contribuir, decidida y responsablemente, aunque dependiente de su naturaleza. En resumen, el vértice A codifica toda la información intrapersonal.

- El vértice B pone en relación la violencia cultural y la cultura de la paz con el ámbito creativo; es desde cada «ámbito», pues cada uno tiene sus particularidades, desde donde se han de poder diseñar y aplicar mecanismos orientados a la creación de una cultura de paz; el «ámbito» es, pues, la cultura en que surge el hecho creativo, al igual que el hecho violento, $y$, por tanto, el aspecto que cabe modificar cuando se habla de «cultura de paz». Es en el «ámbito» donde se puede llevar a cabo la acción educativa en pro de la paz. El vértice B interpela a la suma, a la puesta en común de las singularidades, para obtener una visión global del asunto: en tal sentido se puede decir que identifica los aspectos multipersonales.

- El vértice C establece una correspondencia entre la violencia estructural y la paz positiva y el «campo» de la creatividad, pues son precisamente los elementos del campo aquellos que pueden ejercer de reguladores internos para la consecución del fin de una violencia que es «la suma de todos los choques incrustados en las estructuras sociales y mundiales, y cementados, solidificados, de tal forma que los resultados injustos, desiguales, son casi inmutables» (Galtung, 1998, p.16). El sistema de arbitraje que proporciona el «campo», con las redes de alcance internacional sobre las que se basa y funciona, exalta los aspectos interpersonales. No en vano, Galtung también identifica esta paz positiva con una idea de justicia social (1969, p.183).

¿Cómo trasladar esta propuesta a la teoría (que no la práctica) educativa? Una manera de hacerlo es relacionar cada vértice del triángulo abstractivo con ciertas destrezas que

8 La RAE define abstractivo como «que abstrae o tiene virtud para abstraer». En este sentido se usa en este trabajo: un triángulo que abstrae la esencia de los demás triángulos vistos. 
se puedan adquirir en el aula y que ayuden a trasladar a esta el concepto positivo de paz. Según Hicks, tales destrezas pueden ser afirmación, comunicación y cooperación (1993, pp.99-104):

- la afirmación como asimilación consciente del valor propio, tanto actual como potencial, del individuo. Implica, por ejemplo, la aceptación de la crítica constructiva para mejorar. En el esquema de este trabajo, la afirmación corresponde al vértice A;

- la cooperación es el espíritu de trabajo en pro de la comunidad, con el fin de alcanzar una meta común. Si este objetivo responde al interés propio, la cooperación se diluye en competición. Al enfatizar las relaciones multipersonales, la cooperación corresponde al vértice $B$;

- la comunicación es vista como proceso estructural de interacción con el otro, sin el cual no es posible el proceso. Se entiende como un acto de habla pero también de escucha y de comprensión. Se engloban aquí actitudes de empatía hacia el otro. Por atender a relaciones interpersonales, la comunicación corresponde al vértice $\mathrm{C}$ del triángulo abstractivo.

Esta perspectiva basada en el triángulo abstractivo refuerza una imagen inclusiva de las tradiciones educativas: no se trata de desechar propuestas, sino de construir puentes entre diferentes maneras de entender la educación. El triángulo abstractivo también pone en valor conjunto los aspectos positivos de las grandes tradiciones que Rex Walford identifica en las ideologías educativas (Hicks, 1993, p.29):

- el vértice A corresponde a la tradición centrada en el niño, que intenta potenciar su desarrollo integral en cuanto tal;

- el vértice B recoge el acento en la tradición que se interesa en la instrucción del individuo, por la que este, a partir de la herencia cultural, se prepara para enfrentarse a situaciones predefinidas. En el esquema de Walford se funden aquí las tradiciones liberal humanitaria y utilitaria;

- el vértice $\mathrm{C}$ identifica aspectos de la tradición reconstruccionista, pues ve en la educación un medio para los cambios sociales.

El triángulo abstractivo es un esquema que permite asociar propiedades comunes a diferentes niveles, condensados en tres aspectos (relación personal, interpersonal, multipersonal) con el fin de aglutinar las estrategias orientadas a una mejor implementación práctica de las soluciones creativas que se puedan dar al problema de una Educación para la Paz efectiva. Ha permitido relacionar el concepto de creatividad propuesto por el Modelo de Sistemas con las nociones de paz sin perder de vista el lugar común de tal encuentro, es decir, la Educación para la Paz.

\section{Conclusiones}

A la luz de lo expuesto en este estudio, y a modo de recapitulación, se puede llegar a las siguientes conclusiones:

1. Dado que la creatividad está muy presente en todos los ámbitos de la vida actual, es natural preguntarse por el papel que puede jugar en la educación. 
2. Si, como hacen algunos autores, se aplica la creatividad en distintos aspectos de la acción violenta, entonces se ha de poder aplicar también para la consecución de la paz; en particular, en el ámbito de la Educación para la Paz. De hecho, la historia marca ciertos paralelismos de los que se concluyen potenciales relaciones entre paz y creatividad.

3. Existen varias propuestas sobre lo que es la creatividad, que inciden en que la creatividad no es una cualidad reservada a los genios, sino que cualquier persona está preparada para orientar su creatividad en pro de la paz.

4. Esta deducción del apartado anterior se puede justificar a partir del esquema teórico de Galtung, los triángulos de la violencia y de la paz, relacionándolos vértice a vértice con el triángulo que se infiere de la definición de creatividad de Csíkszentmihályi.

5. La relación entre todos los triángulos se puede condensar en un triángulo esencial o abstractivo, que puede servir de punto de partida para futuros análisis efectivos de la teoría educativa. La propuesta de este trabajo es puramente esquemática, un enfoque orientado a dar una descripción compacta de estrategias que permitan mejorar la implementación práctica de soluciones creativas al problema de la Educación para la Paz en el aula.

\section{Referencias}

Adorno, Th. W. (1998), Educación para la emancipación, Madrid: Morata.

Adorno, Th. W., Horkheimer, M. (2009), Dialéctica de la Ilustración, Madrid: Trotta.

Amabile, T. (2000), «Cómo matar la creatividad», en: Amabile, T. (ed.): Creatividad e innovación, Bilbao: Ediciones Deusto, pp. 1-31.

Arendt, H. (1971), «Thinking and moral considerations», Social Research, 38(3), pp. 417-446.

Arendt, H. (2015), Crisis de la República, Madrid: Trotta.

Barrena, S. (2007), La razón creativa, Madrid: RIALP.

Benito Martínez, J., García Martínez, A. (2001), «Educación para la paz y cultura de la paz», Anales de Pedagogía 19, pp. 65-86.

Bergson, H. (2005), Creative Evolution, New York: Cosimo Classics.

Brunk, C. (2000), «Shaping a vision: The nature of peace studies», en: Fisk, L., Schellenberg, J. (ed.): Patterns of conflict: Paths to peace, Ontario, Canadá: Broadview, pp. 11-34.

Cortina, A. (2008), La Escuela de Fráncfort. Crítica y utopía, Madrid: Síntesis.

Csíkszentmihályi, M. (1996), Creativity. Flow and the psychology of discovery and invention. New York: Harper Collins.

Fernández Mallo, A. (2018), Teoría general de la basura, Barcelona: Galaxia Gutenberg.

Freire, P. (1970), Pedagogía del oprimido, España: Siglo XXI.

Galtung, J. (1969), «Violence, Peace, and Peace Research», Journal of Peace Research 6(3), pp. 167-191.

Galtung, J. (1990), «Cultural violence», Journal of Peace Research 27(3), pp. 291-305.

Galtung, J. (1998), Tras la violencia, 3R: reconstrucción, reconciliación, resolución, Bilbao: Bakeaz-Gernika gogoratuz.

Goldberg, E. (2019), Creatividad. El cerebro humano en la era de la innovación, Barcelona: Crítica.

Hadamard, J. (1954), The Psychology of Invention in the Mathematical Field, New York: Dover. 
Harris, I. (2002), «Conceptual Underpinnings of Peace Education», en: Salomon, G., Nevo, B. (ed.): Peace Education, Mahwah, New Jersey: Lawrence Erlbaum Associates, pp. 15-25. Hicks, D. (1993), Educación para la paz, Madrid: Morata.

Janik, A., Toulmin, S. (2017), La Viena de Wittgenstein, Sevilla: Athenaica.

Lipman, M. (1988), Philosophy goes to school, Philadelphia: Temple University Press.

Lledó, E. (2010), El concepto «poíesis» en la filosofía griega. Heráclito, sofistas, Platón, Madrid: Dykinson.

Martínez Guzmán, V. (2001), Filosofía para hacer las paces, Barcelona: Icaria.

Meyer, S. (2005), «Introduction: Whitehead Now», Configurations 13(1), pp. 1-33.

Pascale, P. (2005), «¿Dónde está la creatividad? Una aproximación al modelo de sistemas de Mihaly Csikszentmihalyi», Arte, Individuo y Sociedad 7, pp. 61-84.

Piobetta, J.-B. (2017), «Juan Amós Comenio (1592-1670)», en: Château, J. (ed.): Los grandes pedagogos, México: Fondo de Cultura Económica, pp. 127-142.

Poincaré, H. (2018), La invención matemática. Cómo se inventa: el trabajo del inconsciente, Oviedo: KRK.

Robinson, K. (2009), El elemento, Barcelona: Random House-Mondadori.

Safranski, R. (2018), Romanticismo, Barcelona: Tusquets.

Sátiro, A. (2002), «¿Crear? Un artículo para dialogar», en: Lipman, M. (ed.): Filosofía y Educación, Madrid: Ediciones de la Torre, pp. 145-161.

Wagensberg, J. (2019), Teoría de la creatividad, Barcelona: Tusquets.

Wallas, G. (1926), The art of thought, London: Jonathan Cape.

Wittgenstein, L. (1980), Culture and value, Oxford: Basil Blackwell.

Wittgenstein, L. (1988), Investigaciones filosóficas, Barcelona: Crítica. 